\title{
A Preliminary Study on qEEG in Burn Patients With Chronic Pruritus
}

\author{
Fiorella K. Miraval, $\mathrm{MD}^{1}$, Vivian L. Shie, $\mathrm{BS}^{2}$, Leon Morales-Quezada, $\mathrm{MD}^{1}$, \\ Carolina Santiago, BA $^{3}$, Bianca Fernandes-Marcondes, PT, $\mathrm{PhD}^{1}$, Deborah Nadler, BA ${ }^{1,2}$, \\ Colleen M. Ryan, MD $^{4,5}$, Jeffrey C. Schneider, MD $^{2}$, Felipe Fregni, MD, PhD, MPH ${ }^{1}$
}

${ }^{1}$ Spaulding Center of Neuromodulation, Spaulding Rehabilitation Hospital, Harvard Medical School, Boston, MA; ${ }^{2}$ Department of Physical Medicine and Rehabilitation, Spaulding Rehabilitation Hospital, Harvard Medical School, Boston, MA; ${ }^{3}$ Brown University, Providence, RI; ${ }^{4}$ Surgical Services, Sumner M. Redstone Burn Center, Massachusetts General Hospital, Harvard Medical School, Boston, MA; ${ }^{5}$ Shriners Hospitals for Children-Boston, Boston, MA, USA

Objective To explore and determine the reorganizational changes in the cortical neural circuits associated with pruritis, this study was undertaken to compare the electroencephalography (EEG) changes in burn patients having primary symptoms of chronic itching (pruritis) and their paired healthy subjects.

Methods Eight subjects were recruited for this exploratory pilot study: 4 patients with pruritus after burn injury matched by gender and age with 4 healthy subjects. EEG recordings were analyzed for absolute alpha, low beta, high beta, and theta power for both groups.

Results The mean age of the burn patients was 41.75 years; while the mean age for the matched healthy subjects was 41.5 years. All subjects were male. A decreased alpha activity was observed in the occipital channels $(0.82 \mathrm{vs}$. 1.4; $\mathrm{p}=0.01)$ and a decreased low beta activity in the frontal area ( 0.22 vs. $0.4 ; \mathrm{p}=0.049)$ in eyes closed conditions. An overall decreased theta trend was observed in both the eyes open and eyes closed conditions in burn patients, compared to healthy individuals.

Conclusion This preliminary study presents initial evidence that chronic pruritus in burn subjects may be associated with brain reorganizational changes at the cortical level characterized by an EEG pattern.

Keywords Burns, Electroencephalography, Pruritus, Brain waves, Quality of life

\footnotetext{
Received July 30, 2015; Accepted November 20, 2015

Corresponding author: Felipe Fregni

Spaulding Center of Neuromodulation, Spaulding Rehabilitation Hospital, 96 Thirteenth Street, Charlestown, MA 02129, USA. Tel: +1-617-952-6156, Fax: +1-617-952-6153, E-mail: Fregni.felipe@mgh.harvard.edu

ORCID: Fiorella K. Miraval (http://orcid.org/0000-0001-8749-7897); Vivian L. Shie (http://orcid.org/0000-0002-2043-9294); Leon Morales-Quezada (http://orcid.org/orcid.org/0000-0003-0736-108X); Carolina Santiago (http://orcid.org/0000-0001-6800-8699); Bianca Fernandes-Marcondes (http:// orcid.org/0000-0002-3190-8797); Deborah Nadler (http://orcid.org/0000-0002-9771-6341); Colleen M. Ryan (http://orcid.org/0000-0002-6455-936X); Jeffrey C. Schneider (http://orcid.org/0000-0001-6652-7700); Felipe Fregni (http://orcid.org/0000-0002-1703-7526).
}

(c) This is an open-access article distributed under the terms of the Creative Commons Attribution Non-Commercial License (http://creativecommons.org/ licenses/by-nc/4.0) which permits unrestricted noncommercial use, distribution, and reproduction in any medium, provided the original work is properly cited Copyright $\odot 2017$ by Korean Academy of Rehabilitation Medicine 


\section{INTRODUCTION}

Pain and itching are two major long-term symptoms that occur after burn injury, reported in as high as $70 \%$ of burn survivors with large burn injuries $[1,2]$. These symptoms are significant disabilities in this population, interfering with sleep, disrupting concentration, and impacting other important quality of life elements, such as return to work [3]. Study results have found that as high as $\mathbf{2 8 \%}$ of burn patients are not employed at longterm follow-up [4]. Furthermore, patients with severe burn injury experience multiple long-term sequelae. In a vast majority of cases, additional disorders, such as depression, post-traumatic stress syndrome, and anxiety, also emerge. Pruritus also remains a major problem in the burn population, affecting not only adults, but also children $[5,6]$. The fact that these types of injuries result in long-term impairments demonstrates the need for additional effective treatments. Through further research however, it is possible to address these unmet clinical needs. Current research on pain has begun to address the proper treatment for patients with burn injury [1], but there remains more to be investigated.

Very little is currently known about the mechanism and biological effects of pruritus, thus there has been no effective treatment reported to date. For this reason, the current study aims to investigate cortical reorganizational changes due to the pruritus experienced by some patients with burn injury. Plastic changes in the mammalian brain have been observed following large scale injuries [7]. Specifically, studies in patients with chronic pain have shown evident changes in cortical activity compared to the cortical activity in healthy subjects $[8,9]$. Therefore, it was hypothesized that pruritus in burn patients, similarly to chronic pain, should also lead to maladaptive brain reorganization, that causes changes in cortical activity compared to healthy subjects.

Patients more than 3 weeks from the time of their burn injury, with pruritus, including subacute and chronic pruritus, due to burn injuries were recruited for this study; each was gender and age matched to a healthy subject. Specifically, chronic pruritus was defined as itching persisting for more than 6 weeks [10].

\section{MATERIALS AND METHODS}

\section{Subjects}

Patients more than 3 weeks from the time of their burn injury, with pruritus, including subacute and chronic pruritus, due to burn injuries were recruited for this study; each was gender and age matched to a healthy subject. Specifically, chronic pruritus was defined as itching persisting for more than 6 weeks [10]. Eight subjects were included in the study: 4 patients with burn injury and 4 healthy individuals. Gender and age were matched between patients with burn injury and the healthy controls. Participant inclusion criteria were as follows [11]: (1) aged between 18 and 65 years; (2) no diagnosis of neurological, psychiatric or unstable medical disorders; (3) no personal history of stroke, traumatic brain injury, epilepsy, unexplained loss of consciousness or severe and frequent headaches; (4) no family history of epilepsy; (5) no history of drug or alcohol abuse or dependence; (6) no current use of central nervous system-effective medications; and (7) no history of brain surgery or presence of metallic implants. The exclusion criteria were as follows: (1) any psychiatric disorders that have led to hospitalization within the past 6 months or have indicated signs of suicidal behavior; (2) learning disorders that may affect the ability to complete assessments; (3) a history of other neurological conditions associated with structural anatomical changes (i.e., stroke, brain injury, Parkinson's); (4) or current use of any anti-epileptic medications or dopaminergic medications (including carbamazepine, oxcarbazepine, and phenytoin).

Subjects with burn injuries were selected according to the following criteria: provided informed consent prior to study participation, aged 18 or older, symptoms of moderate to severe itching at the site of burn injury (at least 4 on the visual analogue scale [VAS] during the previous 3 weeks), injury occurred at least 3 weeks prior to enrollment and had been discharged from acute inpatient care. Burn patients could be enrolled in either inpatient rehabilitation and/or outpatient care. The healthy controls were 4 volunteers from the Boston area recruited through posted study ads at universities, public areas and the internet. All participants provided a list of their current medications or drugs, prior to electroencephalography (EEG) assessment. All study procedures were approved by the Partners Human Research Committee. 


\section{Design}

In this exploratory study we recruited 8 subjects: 4 patients with itching after burn injury and 4 healthy subjects, who were matched by age and gender. In one visit, we used the following instruments: 5-D pruritus scale, VAS for itching activity, VAS for sleep (these instruments were used only in the itching subjects), and EEG recordings for all subjects.

(1) 5-D Pruritus Scale: This is a valid and reliable instrument for measuring itching and changes in itching over time. It is used to measure the 5 dimensions of an itch: degree, duration, direction, disability, and distribution.

(2) VAS for itch activity: This is a 0 to 10 scale, where 0 indicates no itch and 10 indicates frequent itching activity. This scale has been previously used in a study investigating treatment of refractory itching in burn patients. For this study, it was used to assess the level of itching activity.

(3) VAS for sleep: This is a 0 to 10 scale where 0 indicates extremely poor quality of sleep and 10 indicates excellent sleep. Chronic disorders can affect the quality of sleep, so the sleep quality in the burn subjects was measured using this scale.

\section{EEG recording and analysis}

\section{EEG recording}

This study was performed in the Laboratory of Neuromodulation, Spaulding Rehabilitation Hospital, Boston, MA, USA. During the EEG recordings, subjects were asked to close their eyes, but remain awake, while sitting still in a chair, staying relaxed in a quiet room for 5 minutes. An EEG recording was then run under the same conditions, but with eyes open for 5 minutes. The EEG data recording was performed with a 64-channel EGI system with the vertex sensor $(\mathrm{Cz})$ as the reference electrode. The impedances of all electrodes were kept below
$40 \mathrm{k} \Omega$ and band pass filter (100-0.3 Hz).

\section{EEG preprocessing}

The recorded EEG data was analyzed using Net Station 4.5 software (Electrical Geodesic Inc., Eugene, OR, USA). First, a band pass filter of $2-30 \mathrm{~Hz}$ was applied. The artifacts sensors (such as, ocular and muscular) were removed after visual inspection of the raw EEG data. The data was recorded using 64 channels but, in this study, the electrodes of interest were divided into three regions: frontal, temporal, and occipital.

\section{EEG analysis}

Power was calculated in 4 bands: theta $(4-8 \mathrm{~Hz})$, alpha (8-12 Hz), low beta (12-21 Hz), and high beta $(21-30 \mathrm{~Hz})$, each under two conditions: eyes closed and eyes open.

\section{Preliminary statistics}

Given the small sample size, only preliminary statistics were run. Analysis was completed using Stata ver. 13 (StataCorp, College Station, TX, USA). The parametric unpaired Student t-test was used to compare means.

\section{RESULTS}

Demographic information and itch activity are summarized in Table 1 . The mean age for burn patients was 41.75 years, while the mean age for the matched healthy subjects was 41.5 years. All patients were male. All subjects with burn injury reported experiencing symptoms of pruritus since the time of injury. Decreased alpha activity was observed in occipital channels ( 0.82 vs. 1.4; $\mathrm{p}=0.01$ ) and a decreased low beta activity in the frontal area ( 0.22 vs. $0.4 ; p=0.049)$ under eyes closed condition. An overall decreased theta trend in both the eyes open and eyes closed conditions was observed in burn patients, compared with healthy individuals. A comparison

Table 1. Demographic information

\begin{tabular}{ccccccccl}
\hline $\begin{array}{c}\text { Patient } \\
\text { no. }\end{array}$ & Age (yr) & Sex & $\begin{array}{c}\text { Time since } \\
\text { injury (yr) }\end{array}$ & $\begin{array}{c}\text { Duration of } \\
\text { itching (yr) }\end{array}$ & $\begin{array}{c}\text { VAS of itch } \\
\text { activity }\end{array}$ & $\begin{array}{c}\text { VAS of } \\
\text { sleep }\end{array}$ & $\begin{array}{c}\text { 5-D pruritus } \\
\text { scale }\end{array}$ & Medications \\
\hline 1 & 54 & M & 50 & 50 & 8 & 2 & 13 & Fluoxetine \\
2 & 35 & M & 1 & 1 & 6 & 3 & 12 & None \\
3 & 54 & M & 2 & 2 & 5 & 4 & 10 & None \\
4 & 24 & M & 3 & 3 & 6 & 6 & 13 & None \\
\hline
\end{tabular}

VAS, visual analog scale. 
Table 2. Absolute power of each bandwidth activity in the eyes closed condition

\begin{tabular}{|c|c|c|c|c|c|c|c|c|c|}
\hline \multirow{3}{*}{ Brain wave } & \multicolumn{9}{|c|}{ Region of brain } \\
\hline & \multicolumn{3}{|c|}{ Frontal } & \multicolumn{3}{|c|}{ Temporal } & \multicolumn{3}{|c|}{ Occipital } \\
\hline & Burn & Healthy & p-value & Burn & Healthy & p-value & Burn & Healthy & p-value \\
\hline Theta $(4-8 \mathrm{~Hz})$ & 0.37 & 0.79 & 0.06 & 0.29 & 0.64 & 0.10 & 0.59 & 0.99 & 0.10 \\
\hline Alpha (8-12 Hz) & 0.47 & 0.71 & 0.12 & 0.40 & 0.61 & 0.08 & 0.82 & 1.40 & $0.01^{*}$ \\
\hline Low beta $(12-21 \mathrm{~Hz})$ & 0.22 & 0.40 & $0.05^{*}$ & 0.21 & 0.36 & 0.08 & 0.36 & 0.58 & $0.045^{*}$ \\
\hline High beta $(21-30 \mathrm{~Hz})$ & 0.20 & 0.21 & 0.87 & 1.66 & 1.67 & 0.92 & 0.26 & 0.26 & 1.00 \\
\hline
\end{tabular}

Similar results were found when patient $\# 1$ was removed from the analysis, as shown in Table 4 . ${ }^{*} \mathrm{p}<0.05$.

Table 3. Absolute power of each bandwidth activity in the eyes open condition

\begin{tabular}{|c|c|c|c|c|c|c|c|c|c|}
\hline \multirow{3}{*}{ Brain wave } & \multicolumn{9}{|c|}{ Region of brain } \\
\hline & \multicolumn{3}{|c|}{ Frontal } & \multicolumn{3}{|c|}{ Temporal } & \multicolumn{3}{|c|}{ Occipital } \\
\hline & Burn & Healthy & p-value & Burn & Healthy & p-value & Burn & Healthy & p-value \\
\hline Theta $(4-8 \mathrm{~Hz})$ & 0.36 & 0.72 & 0.09 & 0.27 & 0.58 & 0.10 & 0.46 & 0.88 & 0.07 \\
\hline Alpha $(8-12 \mathrm{~Hz})$ & 0.37 & 0.52 & 0.20 & 0.36 & 0.47 & 0.31 & 0.54 & 0.84 & 0.19 \\
\hline Low beta $(12-21 \mathrm{~Hz})$ & 0.26 & 0.37 & 0.18 & 0.21 & 0.30 & 0.22 & 0.32 & 0.49 & 0.11 \\
\hline High beta $(21-30 \mathrm{~Hz})$ & 0.26 & 0.19 & 0.27 & 0.18 & 0.14 & 0.27 & 0.23 & 0.20 & 0.46 \\
\hline
\end{tabular}

Similar results were found when patient \#1 was removed from the analysis, as shown in Table 5.

of the absolute power of each bandwidth for healthy controls compared to burn subjects in the eyes closed condition is detailed in Table 2 . Table 3 details similar data but in the eyes open condition. Results for each condition are further divided into frontal, temporal, and occipital regions of the brain. A topographic map comparing the EEG brain wave changes of a burn subject with pruritus and a healthy subject is included in Fig. 1 (patient \#3 and the matched healthy control).

In particular, as the time since injury for patient \#1 is significantly greater than that for other patients, the preliminary EEG analysis was also run excluding patient \#1 to determine whether the time since the burn may have an effect, as seen in Tables 4 and 5; however, no significant differences were found in this analysis.

\section{DISCUSSION}

Chronic pruritus and pain are related to damage or pathological changes in the peripheral nervous system, though its relation to the long-term changes in central nervous system transmission is not well understood [12]. Given the current lack of existing literature addressing this condition, this exploratory study demonstrates the

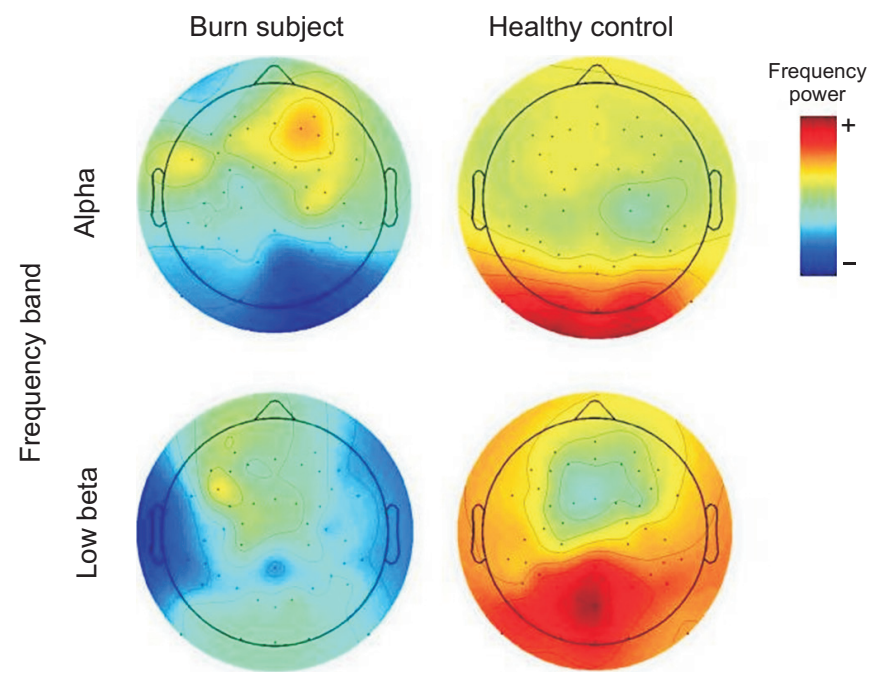

Fig. 1. A topographic map comparing the EEG brain wave changes of the burn subject \#3 and his matched healthy subject.

need to conduct research concerning pruritus. Previous studies have provided evidence of maladaptive brain reorganization in pain by using transcranial magnetic stimulation to assess motor cortex excitability [13]. However, in this current study, focus was placed instead on the changes in the brain waves and assessed by using the 
Table 4. Absolute power of each bandwidth activity in the eyes closed condition (patient \#1 removed from analysis)

\begin{tabular}{|c|c|c|c|c|c|c|c|c|c|}
\hline \multirow{3}{*}{ Brain wave } & \multicolumn{9}{|c|}{ Region of brain } \\
\hline & \multicolumn{3}{|c|}{ Frontal } & \multicolumn{3}{|c|}{ Temporal } & \multicolumn{3}{|c|}{ Occipital } \\
\hline & Burn & Healthy & p-value & Burn & Healthy & p-value & Burn & Healthy & p-value \\
\hline Theta $(4-8 \mathrm{~Hz})$ & 0.33 & 0.72 & 0.1663 & 0.26 & 0.56 & 0.2314 & 0.48 & 0.86 & 0.1607 \\
\hline Alpha $(8-12 \mathrm{~Hz})$ & 0.42 & 0.62 & 0.4067 & 0.37 & 0.56 & 0.3337 & 0.73 & 1.34 & 0.0665 \\
\hline Low beta $(12-21 \mathrm{~Hz})$ & 0.22 & 0.36 & 0.2584 & 0.21 & 0.33 & 0.3174 & 0.37 & 0.57 & 0.2492 \\
\hline High beta $(21-30 \mathrm{~Hz})$ & 0.18 & 0.18 & 0.9321 & 0.16 & 0.15 & 0.8191 & 0.25 & 0.24 & 0.9656 \\
\hline
\end{tabular}

Table 5. Absolute power of each bandwidth activity in the eyes open condition (patient \#1 removed from analysis)

\begin{tabular}{|c|c|c|c|c|c|c|c|c|c|}
\hline \multirow{3}{*}{ Brain wave } & \multicolumn{9}{|c|}{ Region of brain } \\
\hline & \multicolumn{3}{|c|}{ Frontal } & \multicolumn{3}{|c|}{ Temporal } & \multicolumn{3}{|c|}{ Occipital } \\
\hline & Burn & Healthy & p-value & Burn & Healthy & p-value & Burn & Healthy & p-value \\
\hline Theta $(4-8 \mathrm{~Hz})$ & 0.34 & 0.67 & 0.2086 & 0.26 & 0.54 & 0.2495 & 0.48 & 0.82 & 0.2722 \\
\hline Alpha (8-12 Hz) & 0.33 & 0.49 & 0.4172 & 0.31 & 0.46 & 0.4685 & 0.47 & 0.85 & 0.2971 \\
\hline Low beta $(12-21 \mathrm{~Hz})$ & 0.23 & 0.34 & 0.3741 & 0.19 & 0.29 & 0.3940 & 0.33 & 0.49 & 0.3540 \\
\hline High beta $(21-30 \mathrm{~Hz})$ & 0.23 & 0.15 & 0.5337 & 0.16 & 0.12 & 0.6045 & 0.24 & 0.20 & 0.6316 \\
\hline
\end{tabular}

EEG.

This study employed the use of quantitative electroencephalography (qEEG) to analyze the changes in brainwave power. In particular, focus was placed on the alpha, beta and theta brainwave powers. Alpha brainwaves emerge in the awake condition with eyes closed, when the subject is relaxed and processing minimal information. Alpha activity has also been associated with memory recall, lessened discomfort and pain, and reductions in stress and anxiety. Beta brainwaves, on the other hand, signal alertness and attentiveness. A subtype of beta brainwaves, known as low beta or sensorimotor rhythm (SMR) brainwaves, has been associated with mental and emotional disorders, such as depression and insomnia [14]. Finally, theta brainwaves have been found to usually increase during states of deep relaxation and meditation [15]. By comparing the measured cortical activity of patients with pruritus with those of the matched healthy subjects, the study sought to determine the effect of itch at the cortical level.

Preliminary results suggest changes in EEG brainwave patterns related to pruritus. Results indicated a decrease in alpha activity in the occipital area and a decrease in low beta activity in the frontal area during the eyes closed condition in burn patients compared to healthy controls. In addition, an overall decrease in theta activity was observed in both the eyes closed and eyes open conditions in burn patients. These brainwave pattern changes are thus evidence of the maladaptive brain reorganization that occurs in the brains of burn patients after pruritus and/or peripheral damage.

Although alpha activity is widely distributed in the cortex, its function is not clear [16]. However, alpha waves have been found to be associated with meditation and a sense of inner peacefulness created by the release of acetylcholine [17]. In healthy individuals, an increase in alpha is usually observed during the eyes closed conditions, mainly in the posterior occipital-temporal and parietal regions. Conversely, one study, conducted by Jensen et al. [8], demonstrated a decrease in alpha activity and an increase in theta activity in the frontal areas in patients with chronic pain after spinal cord lesion in the eyes closed condition. In this study, results also demonstrated a decrease in alpha activity during the eyes closed condition, but, in contrast, in the occipital region. These study results thus replicate the type of alpha activity seen in chronic pain, but in different areas of the brain.

In this study, a trend, though non significant, was observed in the theta bandwidth. Burn patients with itching recorded a lower theta brainwave power compared to the healthy individuals. Theta brainwaves have been correlated to the reward pathway, especially in the frontal components of the pathway as these frontal regions provide executive control over decisions and reward 
seeking behaviors. The trend of decreased theta activity may be due to the fact that burn patients with itches focus on the itching, thereby causing disruption and distress in their daily activities. Theta waves instigate the release of GABA, a neurotransmitter required to induce relaxation and reduce stress and anxiety, which balances the brain by inhibiting over-excitement [18]. A decrease in theta waves would thus, in turn, decrease the amount of GABA released. The decreased alpha and theta activity may therefore appear as a result of a lack of relaxation $[12,14,19-21]$. However, it is also important to note that one burn participant was taking fluoxetine, an antidepressant. Due to the small sample size, the use of this antidepressant may also account for the decreased trend in the theta oscillations [22].

Analysis of data from the VAS for sleep and quality of sleep items included in the 5-D pruritus scale indicated that most burn patients recruited in this study reported poor quality of sleep, which may affect their brain reorganization. Specifically, a decrease in theta brainwave activity has been observed in insomniac patients. Many pain studies have suggested changes in alpha and theta activity with disruption to the thalamic-cortical loop $[8,23,24]$. This disruption, also known as thalamo-cortical dysrhythmia, refers to the abnormal, internally originated low frequency oscillations in the thalamo-cortical network, which disrupts normal connection and communication between these areas. Patients with unsynchronized loops are therefore prone to mental disorders, such as ADD, depression and insomnia. Disruption of the thalamic-cortical loop may thus explain the observed trend of decreased theta brainwaves in burn patients reporting a poor quality of sleep.

Another finding of note is that burn injury patients with pruritus exhibited a significant decrease in low beta brainwaves (12-21 Hz). Low beta brainwaves, also known as SMR brainwaves, allow individuals to feel more present and in the moment. As shown in Egner et al. [14], training of the low beta brainwave band led to significant attention-enhancing effects in healthy subjects. Low SMR, on the other hand, has been correlated with a lack of focused attention, such as that seen in attention deficit disorder. These low beta brainwaves are inhibited by motion, an issue for burn patients who experience the automatic response to relieve their itching sensation [11]. Studies have shown that people who lack sufficient beta activity exhibit signs of depression or insomnia, disorders observed in many burn patients as a result of the negative effects of their injury on their lifestyle.

In the eyes open condition, no differences were observed between the two groups, with the exception of a decrease in the overall theta trend. However, results from the eyes open condition were not the study focus, due to the susceptibility to artifacts.

Many other studies have also abstained from including the eyes open condition in their analysis, given that they had not found any significant changes.

These study results provide early findings that itching is processed differently than pain. The reward circuit that emerges after itching, which may create a different network as shown by a different EEG pattern, can also affect the central processing of pruritus. Itching is also associated with conscious and voluntary control motor responses, and the reward process that ensues is associated with the ventral tegmental area (VTA), nucleus accumbens (NAc), and prefrontal cortex (PFC). Another difference identified between pain and itching is the specified pathway in the anterolateral spinothalamic tract fiber by the wide dynamic range (WDR) neurons. These neurons are mechanically insensitive, have very low conduction velocity, and have a high electrical threshold that does not show spontaneous activity [14]. These differences between the central processing of pain and itching thus demonstrating the need for different forms of treatment to best facilitate efficient recovery of patients with pruritus. Although this study recruited only 4 burn patients, along with their respectively matched healthy controls, significant results were obtained. Further research should still recruit a larger sample size of patients with pruritus post burn injury in order to obtain more accurate results. In addition, the correlation between measures of sleep quality, drowsiness, and emotional states with EEG activity, may also be examined. Furthermore, as the human brain has the capability of reorganization, it is difficult to ascertain whether pruritus occurs as a result of reorganization or peripheral healing [25]. Therefore, a similar study may be run in the future using burn patients without pruritus as a control group.

The results of this study demonstrate a different EEG pattern in patients with pruritus than that observed in patients with pain. Such results thus add further evidence that pruritus may also cause some maladaptive reorga- 
nization and encourage future studies to utilize other forms of recording and brain activity modulation in order to gain insight into the specific mechanism of pruritus at the cortical level. Such research will aim to provide effective treatment plans to improve quality of life outcomes for burn patients experiencing pruritus.

\section{CONFLICT OF INTEREST}

No potential conflict of interest relevant to this article was reported.

\section{ACKNOWLEDGMENTS}

The contents of this manuscript were developed under a grant from the National Institute on Disability, Independent Living, and Rehabilitation Research (NIDILRR Grant No. 90DP0035). NIDILRR is a Center within the Administration for Community Living (ACL), Department of Health and Human Services (HHS). The contents of this manuscript do not necessarily represent the policy of NIDILRR, ACL, HHS, and should not assume endorsement by the Federal Government.

\section{REFERENCES}

1. Schneider JC, Harris NL, El Shami A, Sheridan RL, Schulz JT 3rd, Bilodeau ML, et al. A descriptive review of neuropathic-like pain after burn injury. J Burn Care Res 2006;27:524-8.

2. Vitale M, Fields-Blache C, Luterman A. Severe itching in the patient with burns. J Burn Care Rehabil 1991; 12:330-3.

3. Schneider JC, Bassi S, Ryan CM. Barriers impacting employment after burn injury. J Burn Care Res 2009; 30:294-300.

4. Mason ST, Esselman P, Fraser R, Schomer K, Truitt A, Johnson K. Return to work after burn injury: a systematic review. J Burn Care Res 2012;33:101-9.

5. Carrougher GJ, Martinez EM, McMullen KS, Fauerbach JA, Holavanahalli RK, Herndon DN, et al. Pruritus in adult burn survivors: postburn prevalence and risk factors associated with increased intensity. J Burn Care Res 2013;34:94-101.

6. Schneider JC, Nadler DL, Herndon DN, Kowalske K, Matthews K, Wiechman SA, et al. Pruritus in pediatric burn survivors: defining the clinical course. J Burn Care Res 2015;36:151-8.

7. Clark SA, Allard T, Jenkins WM, Merzenich MM. Receptive fields in the body-surface map in adult cortex defined by temporally correlated inputs. Nature 1988; 332:444-5.

8. Jensen MP, Sherlin LH, Gertz KJ, Braden AL, Kupper AE, Gianas A, et al. Brain EEG activity correlates of chronic pain in persons with spinal cord injury: clinical implications. Spinal Cord 2013;51:55-8.

9. Boord P, Siddall PJ, Tran Y, Herbert D, Middleton J, Craig A. Electroencephalographic slowing and reduced reactivity in neuropathic pain following spinal cord injury. Spinal Cord 2008;46:118-23.

10. Stander S, Weisshaar E, Mettang T, Szepietowski JC, Carstens E, Ikoma A, et al. Clinical classification of itch: a position paper of the International Forum for the Study of Itch. Acta Derm Venereol 2007;87:291-4.

11. 1997 SSNR (Society for the Study of Neuronal Regulation) Presented Paper Abstracts. J Neuropathy 1997;2: 61-9.

12. Zachariah JR, Rao AL, Prabha R, Gupta AK, Paul MK, Lamba S. Post burn pruritus: a review of current treatment options. Burns 2012;38:621-9.

13. Portilla AS, Bravo GL, Miraval FK, Villamar MF, Schneider JC, Ryan CM, et al. A feasibility study assessing cortical plasticity in chronic neuropathic pain following burn injury. J Burn Care Res 2013;34:e48-52.

14. Egner T, Gruzelier JH. EEG biofeedback of low beta band components: frequency-specific effects on variables of attention and event-related brain potentials. Clin Neurophysiol 2004;115:131-9.

15. Stinson B, Arthur D. A novel EEG for alpha brain state training, neurobiofeedback and behavior change. Complement Ther Clin Pract 2013;19:114-8.

16. de Vries M, Wilder-Smith $\mathrm{OH}$, Jongsma ML, van den Broeke EN, Arns M, van Goor H, et al. Altered resting state EEG in chronic pancreatitis patients: toward a marker for chronic pain. J Pain Res 2013;6:815-24.

17. Lopes da Silva F. Neural mechanisms underlying brain waves: from neural membranes to networks. Electroencephalogr Clin Neurophysiol 1991;79:81-93.

18. Ray WJ, Cole HW. EEG alpha activity reflects attentional demands, and beta activity reflects emotional and cognitive processes. Science 1985;228:750-2.

19. Mendham JE. Gabapentin for the treatment of itching 
produced by burns and wound healing in children: a pilot study. Burns 2004;30:851-3.

20. LaSalle L, Rachelska G, Nedelec B. Naltrexone for the management of post-burn pruritus: a preliminary report. Burns 2008;34:797-802.

21. Casaer M, Kums V, Wouters PJ, Van den kerckhove E, Van den Berghe G. Pruritus in patients with small burn injuries. Burns 2008;34:185-91.

22. Kudina TA, Sudnitsyn VV, Kutyreva EV, Kichigina VF. The serotonin reuptake inhibitor fluoxetine suppresses theta oscillations in the electroencephalogram of the rabbit hippocampus. Neurosci Behav Physiol 2004;34:929-33.
23. Sarnthein J, Stern J, Aufenberg C, Rousson V, Jeanmonod D. Increased EEG power and slowed dominant frequency in patients with neurogenic pain. Brain 2006;129:55-64.

24. Vuckovic A, Hasan MA, Fraser M, Conway BA, Nasseroleslami B, Allan DB. Dynamic oscillatory signatures of central neuropathic pain in spinal cord injury. $J$ Pain 2014;15:645-55.

25. Pons TP, Garraghty PE, Ommaya AK, Kaas JH, Taub E, Mishkin M. Massive cortical reorganization after sensory deafferentation in adult macaques. Science 1991; 252:1857-60. 